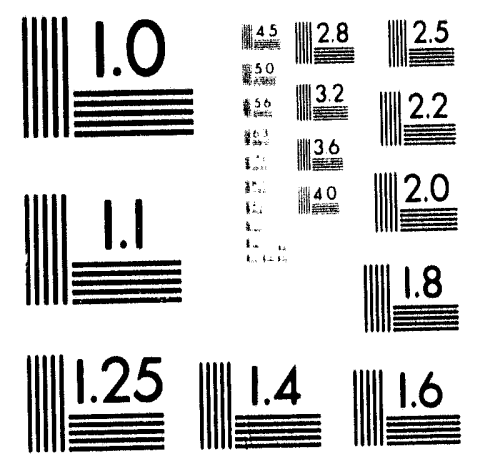



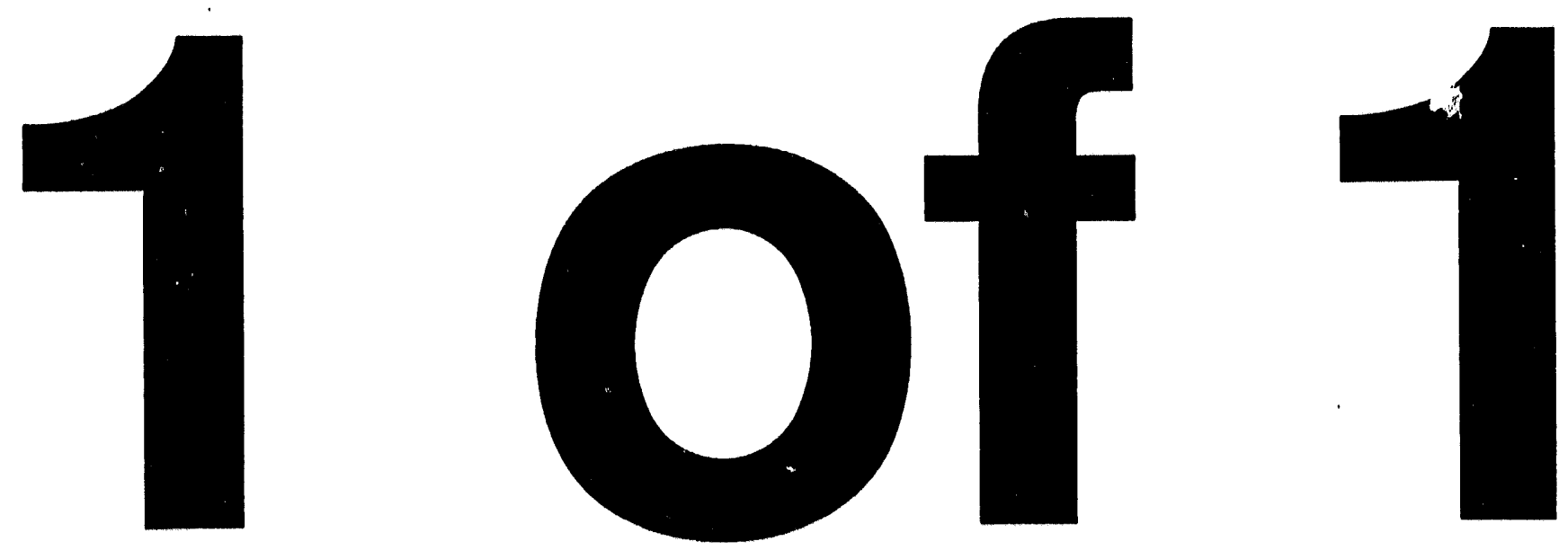
INFRARED FEL PHOTOCHEMISTRY: MULTIPLE-PHOTON DISSOCIATION OF FREON GAS

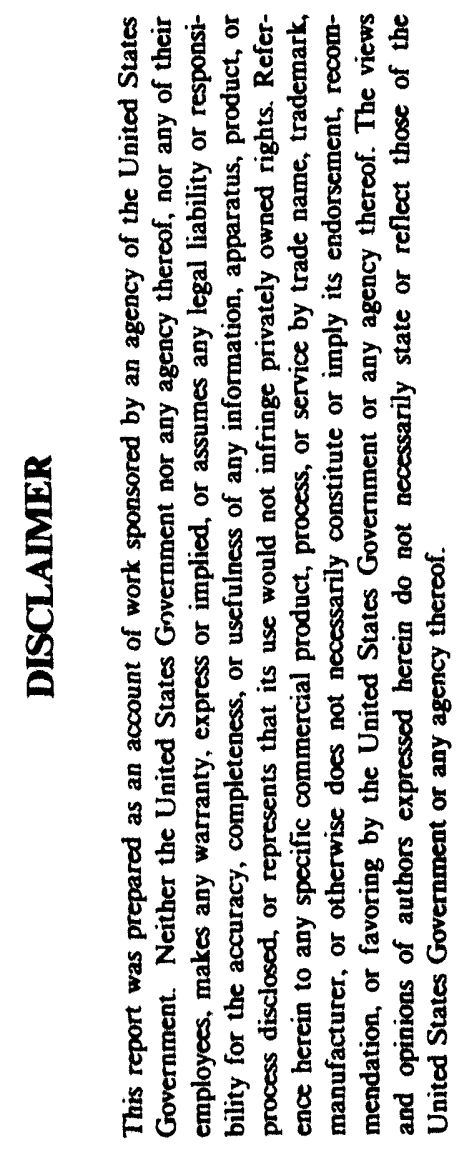

Submitted to:

Brian E. Newnam

James W. Early

John L. Lyman

\section{WEIVLO \\ OCT 072093 \\ OSTI}



Los Alamos National Laboratory, an attirmative action/equal oppontunity employer, is operated by the University of Calitornia for the U.S. Department of Energy under contract W.7405-ENG.36. By acceptance of this article, the publisher recognizes that the U.S. Government relains a nonexclusive, royalty-free license 10 publish or reproduce the published form of this contribution, or to allow others to do so, for U.S. Government purposes. The Los Alamos National Laboratory requests that the publisher identity this anticle as work pertormed under the auspices of the U.S. Department of Energy.

$$
\begin{array}{r}
\text { Form No } 836 \text { R5 } \\
\text { ST } 26291091
\end{array}
$$




\title{
Infrared FEL photochemistry: Multiple-photon dissociation of Freon gas *
}

Brian E. Newnam, James W. Early, and John L. Lyman

Los Alamos National Laboratory, Chemical and Laser Sciences Division, MS J564, Los Alamos, New Mexico 87545 USA

\begin{abstract}
Wavelength tunability, synchrotron sidebands, and picosecond pulse structure are inherent FEL characteristics that should be advantageous for photochemistry involving infrared multiple-photon photodissociation. Tuned to an absorption resonance, the FEL sideband structure will overlap the broad, excited-state spectral absorption and should lead to enhanced dissociation. The LOS Alamos APEX FEL was operated with and without sidebands to test this hypothesis on $\mathrm{CFCl}_{3}$ (Freon 11), an inert chlorofluorocarbon widely used in refrigeration systems and one of the gases implicated as depleting the ozone in the Earth's stratospheric layer. The FEL wavelength was set at the C-Cl stretch absorption resonance at $11.8-\mu \mathrm{m}$, the oscillator cavity length was detuned first to minimize and then to maximize the spectral bandwidth, and the beam was focused through a pair of test cells (1.0 Torr Freon +1.7 Torr air). Comparison of final and initial absorbance spectra indicated the $\mathrm{CFCl}_{3}$ photodissociation yield was $1.2 \%$ for the cell exposed with sideband spectra (3\% FWHM) and 9-ns micropulse separation. Negligible effect was seen without sidebands, albeit at lower total beam fluence. Although the result of this single experiment is not large enough to be conclusive, it does provide a basis for optimizing the FEL temporal and spectral parameters to attain higher photodissociation yield in future tests.
\end{abstract}

"Work supported by the U. S. Dept. of Defense Strategic Defense Initiative Organization under the auspices of the U.S. Department of Energy. 


\section{Introduction}

The man-made chlorofluorocarbons (CFC), especially Freon $11\left(\mathrm{CFCl}_{3}\right)$ and Freon $12\left(\mathrm{CF}_{2} \mathrm{Cl}_{2}\right)$, have attracted public attention because they have been linked to depletion of the ozone layer in the Earth's stratosphere, resulting in increased surface exposure to UV-B radiation $(294-320 \mathrm{~nm})$ [1-4]. Freons released at the surface of the Earth eventually reach the stratosphere where exposure to sunlight below $200 \mathrm{~nm}$ dissociates the chlorine atoms. Each free chlorine atom then catalytically removes an oxygen atom from up to 100,000 ozone molecules before leaving its itinerant cycle. The 60 to 140 year life of Freons as they diffuse through the troposphere, as well as the continuing new releases by human processes, have prompted an international agreement known as the Montreal Protocol on Substances that Drsplete the Ozone Layer-1987 [1-3], amended in London in 1990 [2], to curtail further production by 1996.

Thomas Stix [5] of Princeton University has proposed an atmospheric engineering solution to the CFC problem that would use over one-thousand, 2 to 3-MW infrared lasers stationed around the globe to photodissociate the Freon molecules within the troposphere, before they drift into the stratosphere. The freed chlorine atoms would then combine with water or oxygen and fall to the Earth in rain. This approach is based on multiple-photon absorption of 30 to 40 infrared photons at the $\mathrm{C}-\mathrm{Cl}$ (or C-F) stretch resonant wavelengths between 8 to $12 \mu \mathrm{m}$ (specific to the type of Freon). Laboratory demonstrations with nanosecond-pulse infrared lasers have shown that 30 to 40 absorbed photons can release the $\mathrm{Cl}$ atom with sufficient efficiency to be of potential interest [6]. Even so, Stix' projections for the scope and cost of such a laser solution are not considered feasible with present technology, perhaps by a factor of 10 to 20 [7]. Bullock, et al. [8] have suggested that an optimization of the laser pulselength and spectrum could significantly reduce the size of Stix' proposal.

Free-electron lasers offer the versatility in spectral and temporal output that may enable, via the multiple-photon absorption process, attainment of efficient 
photodissociation of Freons, as well as other classes of large molecules. FELs offer easy wavelength tunability to molecular absorption resonances, adjustable spectral bandwidth by synchrotron sidebands or frequency chirping, high-peak power in picosecond pulse trains, and potential scalability to high-average power levels. Synchrotron sideband emission, a feature unique to FELs, manifests as an irregular lasing spectrum on the long wavelength side of the resonant wavelength. For example, the Los Alamos APEX FEL has operated with sidebands extending out to $10 \%$ beyond the initial lasing line.

We briefly describe the salient features of the multiple-photon absorption (MPA) processes: Initially, radiation within a specific, narrow-band infrared wavelength is required to excite a molecule up the ladder of discrete energy levels into the quasicontinuum of states. The absorption spectrum of these excited molecules is shifted to wavelengths longer than the initial resonance by up to $10 \%$. Subsequent exposure within this broad wavelength range call further excite the molecule past the dissociation level. This is made possible by the monotonically increasing density of available states within the quasi-continuum with increasing energy level [9]. Generally, MPA depends primarily on laser fluence with a weak dependence on intensity of laser excitation (incoherent addition of laser photons is sufficient) [10]. However, for $\mathrm{CF}_{2} \mathrm{HCl}$ (a molecule similar to the Freons), high-intensity mode-locked $\mathrm{CO}_{2}$ laser pulses have produced 5-10 faster dissociation rates than non-mode-locked pulses of the same fluence [11].

We envisioned that the FEL sideband structure could match the broad, excitedstate spectral absorption and lead to greater dissociation efficiency than with single, narrow-band wavelength. Supporting this expectation are previous ir multiple-photon dissociation experiments with other heavy molecules, e.g. $\mathrm{CF}_{2} \mathrm{HCl}[12]$ and $\mathrm{OsO}_{4}$ [13] in which exposure with two wavelengths produced 10 to 300 more dissociated products than with a single, resonant wavelength. To test this hypothesis, we tuned 
the Los Alamos APEX FEL to the 11.8- $\mu \mathrm{m}$ absorption resonance (C-Cl stretch) of Freon 11 and exposed a pair of test cells, with and without sidebands. The following sections (2-5) present the experimental procedures, the experimental results, a discussion of the results, and a summary including suggestions for future tests.

\section{Experimental procedures}

Two identical test cells ( $76 \mathrm{~mm}$ length, $35 \mathrm{~mm}$ diameter) with $\mathrm{NaCl}$ windows were filled with 1.0 Torr of $\mathrm{CFCl}_{3}$ plus 1.7 Torr or air to provide $\mathrm{H}_{2} \mathrm{O}$ and $\mathrm{O}_{2}$ known to be effective scavengers for photodissociated $\mathrm{Cl}$ atoms [6]. The infrared transmission of the gas-filled cells was measured with a Mattson Instruments FTIR spectrometer set for $0.5 \mathrm{~cm}^{-1}$ resolution and equipped with computer data processing capability for storing and processing successive measurements.

The FEL beam was focused at the center of the cells to a spot-size radius of 0.35 $\mathrm{mm}$ as measured with a Spiricon linear pyroelectric array. Laser energy was monitored at the entrance and exit of the cells with Laser Precision pyroelectric energy meters. The FEL was operated at $1 \mathrm{~Hz}$ with parameters listed in Table 1 , and the temporal dependence of the macropulse and integrated spectrum were monitored on each shot. The degree of sideband generation was obtained by adjusting the resonator length, attaining bandwidths of $1 \%$ (full-width at $1 / \theta$ ) when detuned and $3 \%$ with zero detuning (see Fig. 1).

We conducted tests at the strongest absorption resonance of $\mathrm{CFCl}_{3}$ at $11.8 \mu \mathrm{m}$, corresponding to a $\mathrm{C}-\mathrm{Cl}$ stretch vibration. Figure 2 shows a comparison of the initial $\mathrm{CW}$ transmittance measured with the FTIR spectrometer and the pulsed transmittance using the FEL tuned for narrow bandwidth $(\sim 1 \%)$ and attenuated by a factor of 100 to $\sim 1 \mathrm{MW} / \mathrm{cm}^{2}$ peak intensity. Even at this relatively low intensity, the absorption resonance is significantly saturated. Subsequently, the cells were exposed to 2000 
macropulses of the unattenuated FEL beam tuned to $11.8 \mu \mathrm{m}$. Visual observation verified the absence of optical breakdown within the cells. At the end of the FEL exposures, the absorbance spectra were remeasured and compared to the initial absorbance to determine the fractional dissociation.

Regrettably, the very limited availability of the FEL beam forced us to severely abbreviate the scope of our tests planned for a wide range of FEL and Freon gas parameters. Thus, only two exposures were accomplished, with and without sidebands, but with different micropulse separations and pulse fluences.

\section{Experimental results}

Figure 3 shows the final absorbance and net change in absorbance of a cell of Freon 11 measured after exposure to $2100 \mathrm{FEL}$ macropulses with $3 \%$ (FW-1/e) sideband spectra and 9-ns micropulse separation (se日 Table 1). Since absorbance is proportional to molecular number density, fractional photodissociation is derived directly from the ratio of the absorbance change and absorbance spectrum measured just prior to exposure. The most accurate measure of fractional absorbance change at each of the two resonances $(9.26 \mu \mathrm{m}$ and $11.78 \mu \mathrm{m})$ is obtained from the ratio of the absorbance change-wave number product integrated over the shaded regions divided by the initial absorbance integrated over the same spectral ranges. A small, but definite photodissociation yield of $1.2 \%$ was so derived (see Table2). Previous photodissociation studies by Hudgens [14] identify this photodissociation process as $\mathrm{CFCl}_{3} \rightarrow \mathrm{CFCl}_{2}+\mathrm{Cl}$. The test without sidebands (1\% FW-1/e) and lower intensity produced negligible photodissociation product.

The resonant wavelength of the FEL. exhibited significant instability $( \pm 0.07 \mu \mathrm{m})$, comparable to the $0.2 \mu \mathrm{m}$ FWHM bandwidth of the $11.8-\mu \mathrm{m}$ resonance. Likewise, the pulse-to-pulse variation of the laser output energy was large, with $\pm 30-40 \%$ standard deviation. The output power level was $\sim 10 \%$ of that normally produced by this $F E L$, 
due primarily to the high diffraction losses of the particular resonator in place at the time of these tests.

\section{Discussion}

The low dissociation yield obtained in these tests probably involved the following parameters:

1. Laser wavelength: The $\pm 0.07 \mu \mathrm{m}$ pulse-to-pulse variation of the FEL resonant wavelength may have been too large. With greater stability, a more optimal initial wavelength would have been on the long side of the resonance [6], perhaps between 11.9 and $12.0 \mu \mathrm{m}$.

2. Laser fluence and micropulse separation: Efficient use of sidebands requires sufficient fluence at the initial molecular resonance to excite molecules into the quasicontinuum. Our $0.6 \mathrm{~J} / \mathrm{cm}^{2}$ axial fluence at the resonant wavelength, distributed over $25 \mu \mathrm{s}$, was probably insufficient. To offset collisional de-excitation (decay time estimated at 1 to $10 \mu \mathrm{s} /$ Torr) of the molecules during the macropulse, a more compressed pulse structure would be advantageous.

3. Scavenger gas: The number of scavenger molecules $\left(\mathrm{O}_{2}\right.$ and $\left.\mathrm{H}_{2} \mathrm{O}\right)$ available per dissociated $\mathrm{CFCl}_{3}$ molecule was probably insufficient, allowing exciessive recombination to occur. A much larger ratio (10:1) of scavenger molecules per CFC molecule were effective in photodissociation experiments by Lupo and Quack [6].

Our initial plans had been to investigate most of the above parameter variations during these experiments. With more FEL beam time and adjustment of parameters, larger dissociation yields would probably be obtained.

As an alternative to the use of sidebands, negative frequency chirping during the macropulse may be an eifective way to excite molecules up the vibrational levels to dissociation. Such capability has recently been reported by A. van der Meer, et al. [15] using the Dutch FEL at the FOM Institute for Plasma Physics at Nieuwegein. 


\section{Summary}

When operated with spectral sidebands extending 3\% beyond the resonant wavelength and $25-\mu \mathrm{s}$ macropulselength, the Los Alamos APEX FEL photodissociated a small fraction (1.2\%) of the irradiated volume of a cell of Freon 11 (1.0 Torr +1.7 Torr air). Negligible effect was seen without sidebands, albeit at lower total beam fluence. With the influence of a wide range of parameters (FEL temporal and spectral structure, scavenger gas, cell pressure) yet remaining to be explored, the potential advantages of FEL sidebands on multiple-photon dissociation remains an open question. Although the result of the single experiment reported here is not large enough to be conclusive, it does provide a basis for optimizing the FEL temporal and spectral parameters to attain higher photodissociation yield in future tests.

\section{Acknowledgments}

We are grateful for the contributions of a number of Los Alamos personnel including S. Kinkead, who supplied the purified Freons, D. M. Harradine and K. A. Funk for assistance with the FTIR spectrometer, S. Elliott and C. F. Keller for advice on atmospheric chemistry, and to members of the FEL project team including $K$. F. McKenna, S. C. Bender, D. W. Feldman, P. G. O'Shea, and T. J. Zaugg.

\section{References}

[1] Scientific Assessment of Stratospheric Ozone: 1989, Vol. 1, World Meteorological Org., Global Ozone Res. and Monitoring Project- Rpt. No. 20, (World Met. Org., Geneva, 1990).

[2] Scientific Assessment of Ozone Depletion: 1991, World Meteorological Org./United Nations Environment Prog. Rpt., (World Met. Org., Geneva, 1992).

[3] R. Stolarski, R. Bojkov, L. Bishop, C. Zeretos, J. Staehelin, and J. Zawodny, Science 256 (1992) 342. 
[4] Recent Developments in the Ozone Layer and Ultraviolet Radiation over Belgium and the Netherlands, joint report by Belgian Royal Meteorological Inst. [KMI], Dutch Natl. Inst. for Public Health and Environmental Protection [RIVM], and Royal Dutch Meteorological Inst. [KNMI], Aug., 1993.

[5] T. H. Stix, J. Appl. Phys. 66 (1989) 5622.

[6] D. W. Lupo and M. Quack, Chem. Phys. Lett. 130 (1986) 371.

[7] Policy Implications of Greenhouse Warming, (Nat. Academy Press, Wash., D.C., 1992), pp. 457-458.

[8] D. L. Bullock, D. Dee, and M. M. Valley, Proc. SPIE Vol. 1628 (1992) 326.

[9] J. L. Lyman, H. W. Galbraith, and J. R. Ackerhalt, Los Alamos Science 3 (1982) 66. [10] J. L. Lyman, J. W. Hudson, and S. M. Fround, Opt. Commun. 21 (1977) 112.

[11] D. S. King and J. C. Stephenson, Chem. Phys. Lett. 66 (1979) 33.

[12] P. F. Gonzalez-Diaz, M. Santos, C. L. Siguenza, and J. A. Torresano, Laser Chem. 12 (1992) 25.

[13]R. V. Ambartzumian, in Multiple-Photon Excitation and Dissociation of Polyatomic Molecules, C. D. Cantrell, ed., (Springer-Verlag, Berlin, 1986), pp. 133-134.

[14] J. W. Hudgens, J. Chem. Phys. 68 (1978) 777.

[15] A. F. G. van der Meer, P. W. van Amerstoort, R. J. Bakker, and D. Oepts, Nucl. Instr. and Meth., this volume, (1994). 
Table 1

FEL experimental parameters

Without sidebands With sidebands Units

$\begin{array}{lccc}\text { Wavelength, resonant } & 11.8 & 11.8 & \mu \mathrm{m} \\ \text { Spectral bandwidth, FW-1/e } & 1 & 3 & \% \\ \text { Stability of resonant wavelength } & \pm 0.07 & \pm 0.07 & \mu \mathrm{m} \\ \text { Macropulse energy } & 1.7 \pm 0.7 & 13 \pm 4 & \mathrm{~mJ} \\ \text { Beam 1/8 radius at focus } & 0.35 & 0.35 & \mathrm{~mm} \\ \text { Macropulse fluence thru cell } & 0.6 \pm 0.3 & 4.7 \pm 1.6 & \mathrm{~J} / \mathrm{cm}^{2} \\ \text { Number of FEL macropulses } & 2000 & 2100 & \\ \text { Macropulse length } & 25 & 25 & \mu \mathrm{s} \\ \text { Micropulse separation } & 46.2 & 9.2 & \mathrm{~ns} \\ \text { Micropulse energy } & 3.2 \pm 1.3 & 4.8 \pm 1.6 & \mu \mathrm{J} \\ \text { Micropulse length } & 10 & 10 & \mathrm{ps} \\ \text { Micropulse peak power } & 320 \pm 130 & 480 \pm 160 & \mathrm{~kW} \\ \text { Peak intensity } & 115 \pm 50 & 175 \pm 60 & \mathrm{MW} / \mathrm{cm}^{2}\end{array}$

\section{Table 2}

Experimental results

Without sidebands

With sidebands

Macropulse fluence at $11.8 \pm 0.1 \mathrm{fm}$

$\begin{array}{cc}0.6 \mathrm{~J} / \mathrm{cm}^{2} & 0.6 \mathrm{~J} / \mathrm{cm}^{2} \\ \sim 0 & 4 \mathrm{~J} / \mathrm{cm}^{2} \\ 58 \% & 61 \% \\ <0.5 \% & 1.2 \%\end{array}$

Macropulse fluence in sidebands

Fraction of gas cell volume exposed

Per cent dissociation of exposed gas

$<0.5 \%$

$1.2 \%$ 


\section{Figure captions}

Fig. 1. FEL spectrum with sidebands near $11.8 \mu \mathrm{m}$ integrated over one maciopulse. Scale: $0.13 \mu \mathrm{m} / \mathrm{cm}$ with wavelength increasing to the left.

Fig. 2. Transmission at the $11.8 . \mu \mathrm{m}$ resonance of $\mathrm{CFCl}_{3}$ as measured with an FTIR spectrometer (line) and the pulsed FEL (circles). FEL beam was attenuated to an intensity of $\sim 1 \mathrm{MW} / \mathrm{cm}^{2}$ with $1 \%$ bandwidth ( $\mathrm{FW}$ at $1 / \theta$ ). $\mathrm{CFCl}_{3}$ partial pressure: 1.0 Torr partial pressure; path length: $76 \mathrm{~mm}$.

Fig. 3. FTIR absorbance spectrum of $\mathrm{CFCl}_{3}$ (1.0 Torr, $76 \mathrm{~mm}$ path length): a) before exposure, and b) net decrease after FEL exposure with $3 \%$ sidebands. The scale of $b$ ) is magnified by a factor of $70 x$. (Exposure conditions are given in Tables 1 and 2.) 


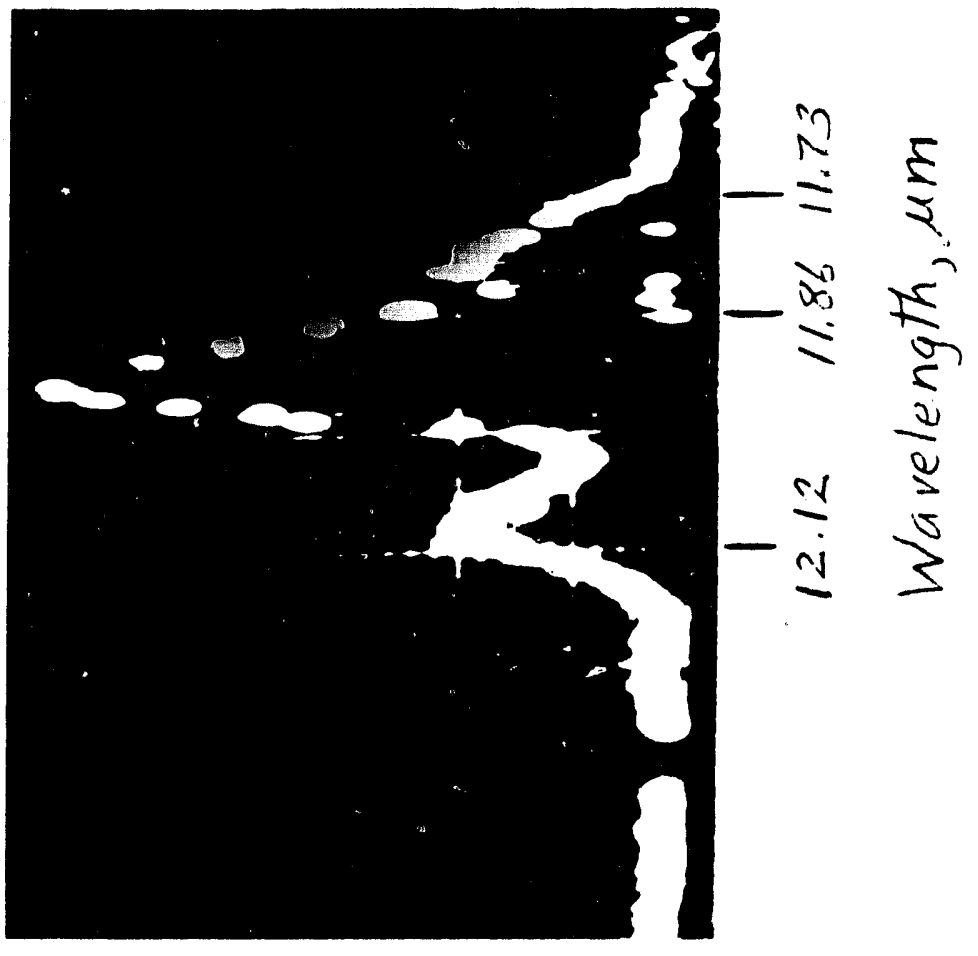




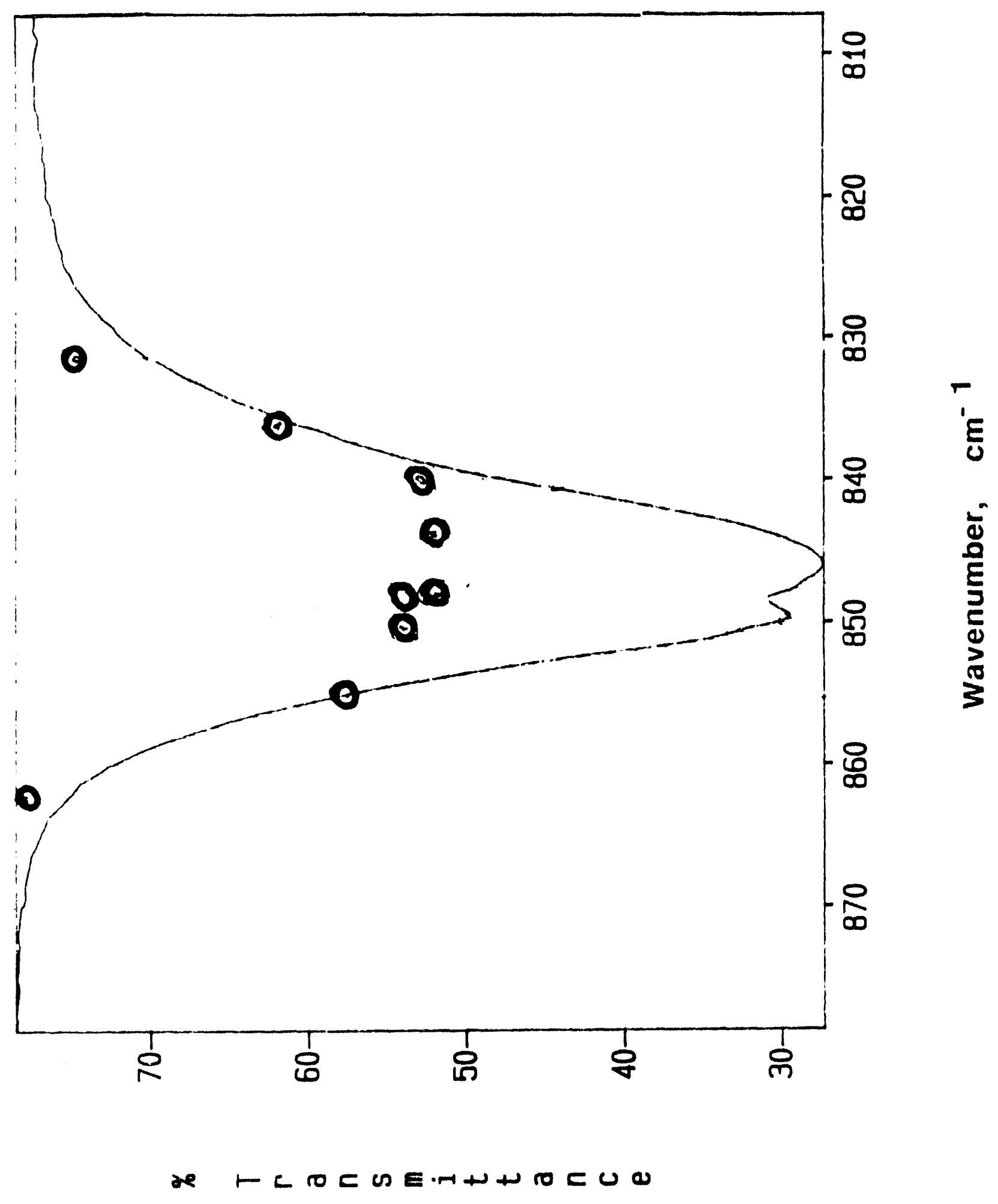

12 


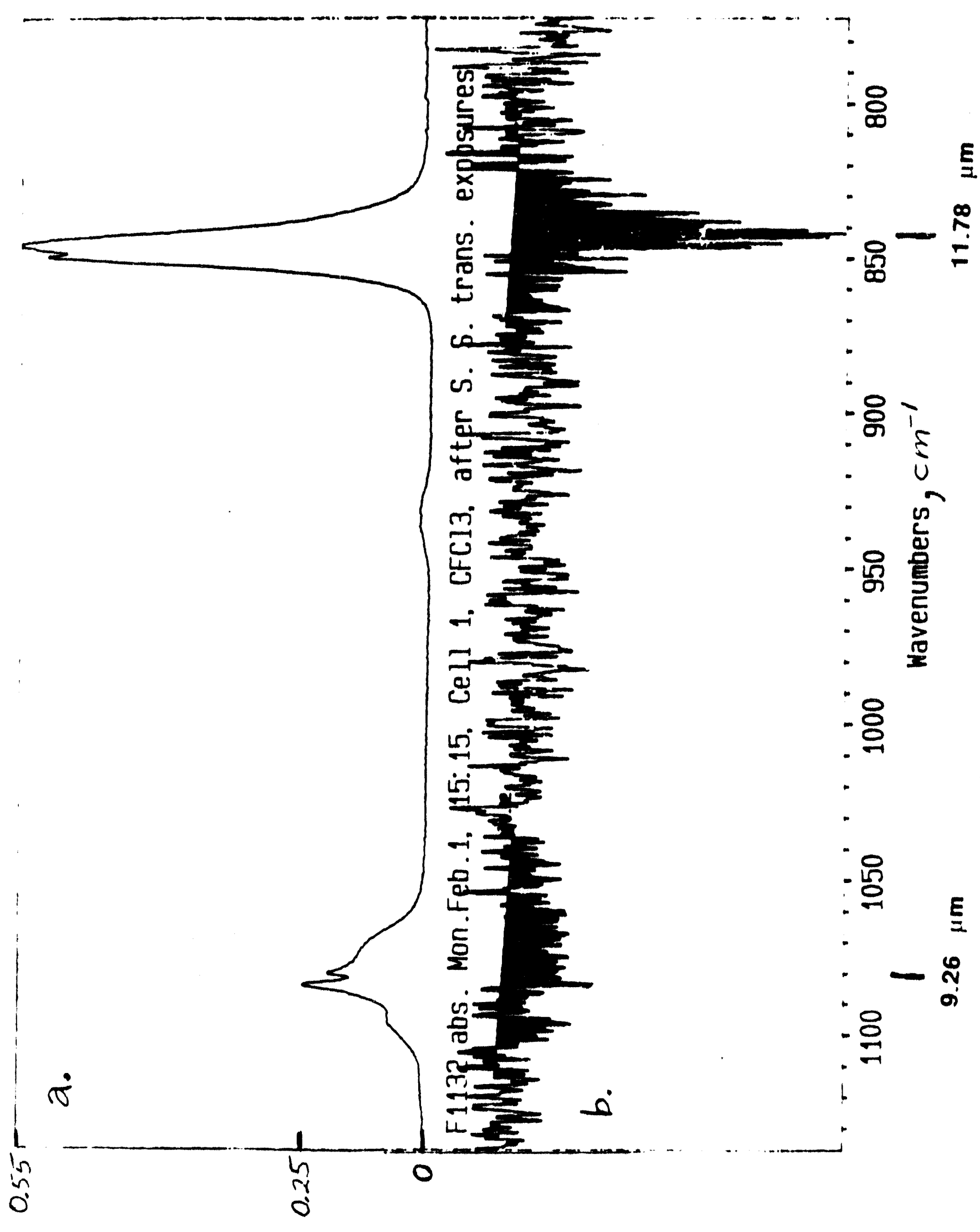

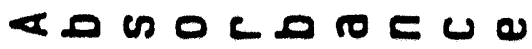

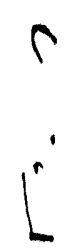



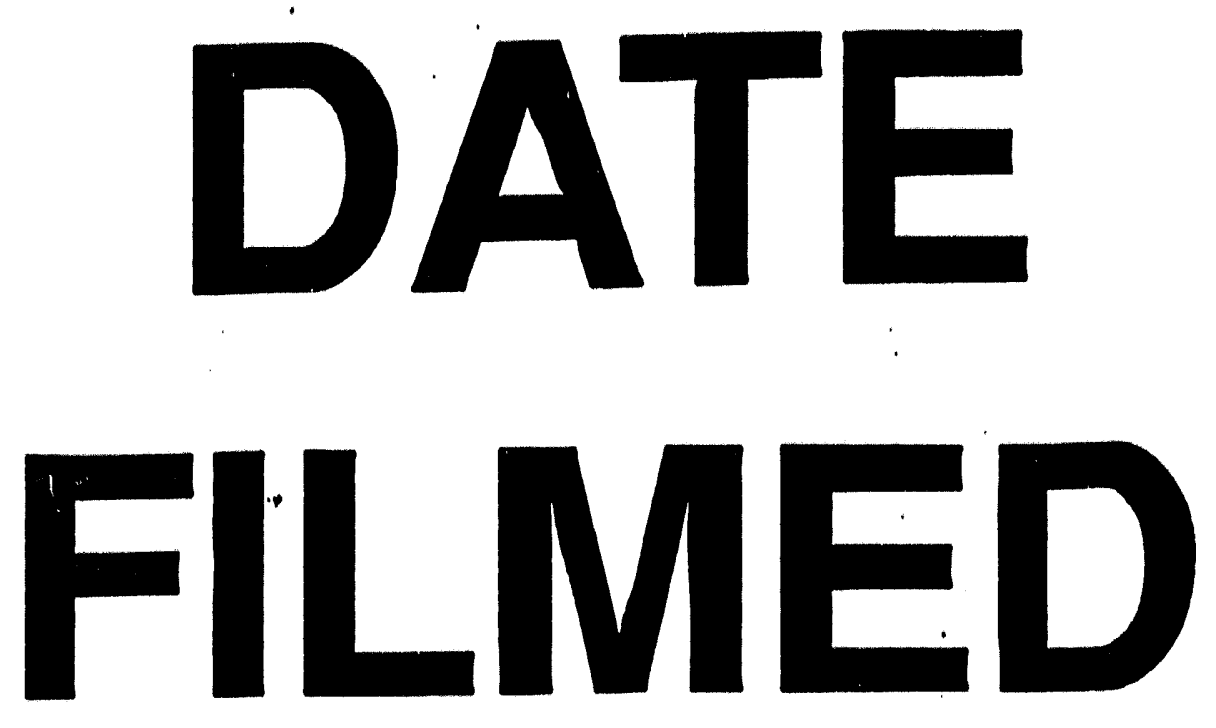

$12 / 6 / 93$
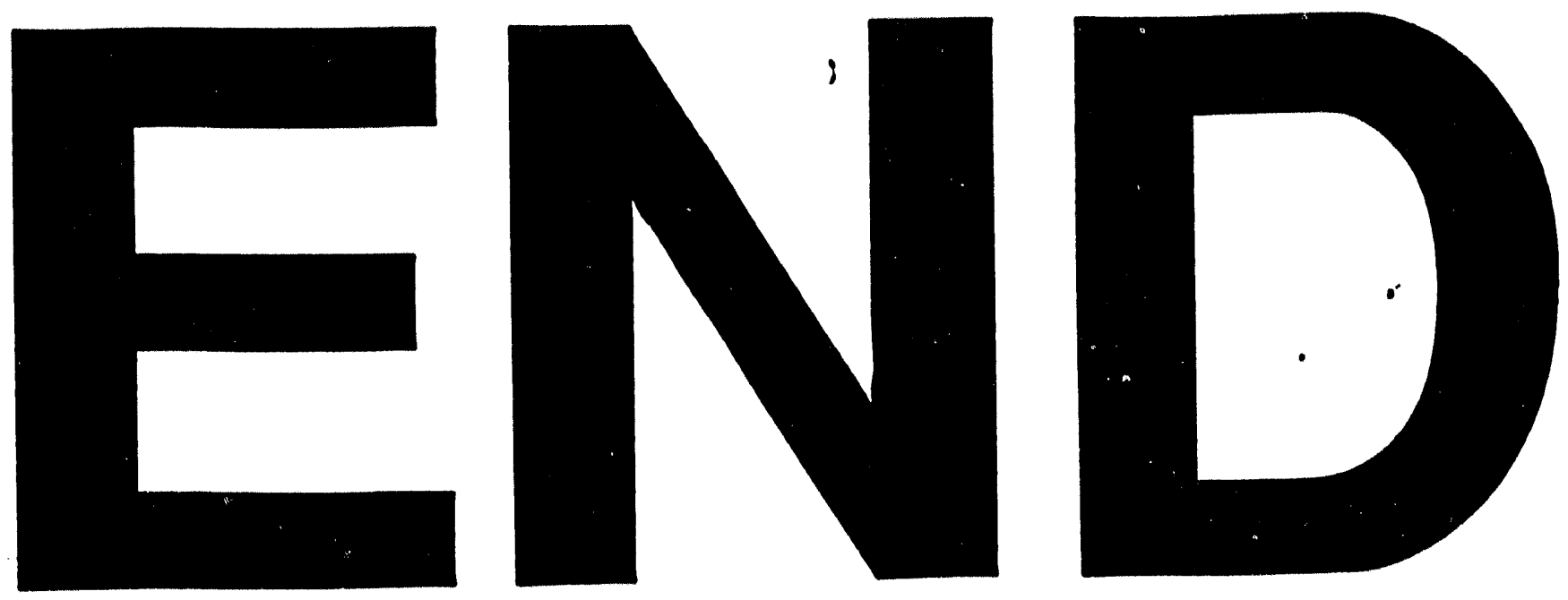
$$
1
$$ 